\title{
IL-15 Deficient Tax Mice Reveal a Role for IL-1 $\alpha$ in Tumor Immunity
}

\author{
Daniel A. Rauch, John C. Harding, Lee Ratner*
}

Department of Medicine, Division of Molecular Oncology, Washington University School of Medicine, Saint Louis, Missouri, United States of America

\begin{abstract}
IL-15 is recognized as a promising candidate for tumor immunotherapy and has been described as both a promoter of cancer and a promoter of anti-cancer immunity. IL-15 was discovered in cells transformed by HTLV-1, the etiologic agent of adult $T$ cell leukemia/lymphoma (ATL) and the human retrovirus that carries the Tax oncogene. We have developed the TAX-LUC mouse model of ATL in which Tax expression drives both malignant transformation and luciferase expression, enabling non-invasive imaging of tumorigenesis in real time. To identify the role of IL-15 in spontaneous development of lymphoma in vivo, an IL-15 tumor growth and accelerated mortality and demonstrated that IL-15 was not required for Tax-mediated lymphoma but was essential for anti-tumor immunity. Further analysis revealed a unique transcriptional profile in tumor cells that arise in the absence of IL-15 that included a significant increase in the expression of IL-1 $\alpha$ and IL- $1 \alpha$-regulated cytokines. Moreover, anti-IL- $1 \alpha$ antibodies and an IL- 1 receptor antagonist (Anakinra) were used to interrogate the potential of IL-1 $\alpha$ targeted therapies in this model. Taken together, these findings identify IL-15 and IL-1 $\alpha$ as therapeutic targets in lymphoma.
\end{abstract}

Citation: Rauch DA, Harding JC, Ratner L (2014) IL-15 Deficient Tax Mice Reveal a Role for IL-1 $\alpha$ in Tumor Immunity. PLoS ONE 9(1): e85028. doi:10.1371/ journal.pone.0085028

Editor: Zhi-Ming Zheng, National Institute of Health - National Cancer Institute, United States of America

Received September 11, 2013; Accepted November 20, 2013; Published January 8, 2014

Copyright: (c) 2014 Rauch et al. This is an open-access article distributed under the terms of the Creative Commons Attribution License, which permits unrestricted use, distribution, and reproduction in any medium, provided the original author and source are credited.

Funding: PHS grants CA94056, CA10073, and CA63417. The funders had no role in study design, data collection and analysis, decision to publish, or preparation of the manuscript.

Competing Interests: The authors declare no competing financial interests.

*E-mail: Iratner@dom.wustl.edu

\section{Introduction}

The Dual Role of IL-15 in Hematopoietic Malignancies

IL-15 is a central cytokine in lymphocyte development, hematopoietic malignancies, and immunotherapy, where it has paradoxically been described as both a promoter of cancer and a promoter of anti-cancer immunity. [1,2] IL-15 belongs to the family of four-helix-bundle cytokines (also including IL-2, IL-4, IL7, IL-9, and IL-21) which use receptors that share a common gamma-c chain and have unique alpha chains. While there is partial redundancy among this family of cytokines, IL-15 has emerged as one particularly suited for antitumor activity. IL-15 is an important factor in the development, homeostasis, proliferation, and activity of $\mathrm{CD}^{+} \mathrm{T}$ cells, NK cells, NKT cells, and intraepithelial $\mathrm{T}$ cells. [3] IL-15 also activates monocytes, macrophages, and dendritic cells; inhibits apoptosis in granulocytes and lymphocytes; promotes a persistent immune response without inducing $\mathrm{T}_{\text {reg }}$ activity; and represents a prime candidate for facilitating innate and durable adaptive tumor immunity. [2].

Although IL-15 is regarded as an excellent candidate for tumor therapy, it has also been characterized as a promoter of cancer. Co-discovered in HuT-102 cells transformed by HTLV-1 [4,5], subsequent studies have confirmed the importance of IL-15 in a variety of hematopoietic malignancies and solid tumors. The potential mechanisms by which IL-15 mediates its pro-tumor activity include protecting tumor cells from apoptosis, and promoting proliferation, migration, invasion and metastasis. $[6,7]$ IL-15 is an important mediator of growth, and survival of the malignant cells in hematopoietic malignancies and solid tumors. $[6,8,9]$ In fact, overexpression of IL-15 in transgenic mice is sufficient to cause CD8 leukemia and T-LGL or NKT leukemia. $[10,11]$ While IL-15 over-expression promotes leukemia/lymphoma, it is less well understood if IL-15 is a necessary prerequisite for cancer development. Similarly, IL-15 has the potential to be a high-value therapeutic target. What is less clear is whether systemic modulation of IL-15 activity represses or stimulates hematopoietic malignancies in vivo.

\section{TAX-LUC Mice as a Model of Human Lymphoma}

HTLV-1, the etiologic agent of adult $\mathrm{T}$ cell leukemia/ lymphoma (ATL), is a human retrovirus that carries the Tax oncogene. Tax activates viral transcription through the $5^{\prime}$ long terminal repeat (LTR) but is also capable of constitutively activating the NFкB pathway in infected cells which results in overexpression of IL-15. [5,12] As a model of ATL, TAX transgenic mice, in which Tax expression is governed by the human granzyme B promoter, develop large granular lymphocytic lymphoma. [13] The TAX-LUC strain is a second generation model developed to take advantage of Tax as a strong activator of viral transcription through the HTLV-1 LTR. [14] A transgene in which firefly luciferase is driven by the HTLV-1 LTR (LTR-LUC) was introduced to make double transgenic TAX-LUG mice. Therefore, in TAX-LUG mice, Tax expression drives both tumorigenesis and luciferase expression, which can be detected non-invasively using bioluminescence imaging. Lymphoma in this model presents as subcutaneous tumors, massive splenomegaly, involvement of the bone marrow resulting in hypercalcemia and osteolytic bone lesions, and a chronic inflammatory response 
involving the persistent activation and recruitment of neutrophils to the tumor. $[14,15]$ Within the tumors, the malignant lymphoma cells comprise only $10-15 \%$ total tumor cells. The majority of the tumor is populated by infiltrating immune cells, primarily neutrophils, but also $\mathrm{T}$ cells, NK cells, and macrophages. Like ATL patient samples and cell lines, IL-15 mRNA is expressed in tumors that arise in TAX-LUC mice along with other NFкB inducible genes. [16] While IL-15 expression is associated with Tax tumor initiation and growth, the net effect of IL-15 expression in vivo has not yet been established.

\section{What is the Net Effect of IL-15 in Tax-lymphoma?}

Using Tax-LUC mice as a model in which IL-15 is capable of promoting both cancer and cancer immunity, we sought to determine the effect of IL-15 loss on tumor growth in vivo. To this end an IL-15 ${ }^{-/-}$TAX-LUC strain was developed and examined. Based on our initial observation that IL-15 overexpression correlated with tumor growth we hypothesized that loss of IL-15 would impede tumor onset or growth. Surprisingly, IL-15 deficient TAX-LUC mice developed significantly larger tumors compared to IL- $15^{+/+}$TAX-LUC littermates. Further analysis of these tumors revealed effects of IL-15 loss on both the malignant population and the tumor infiltrating immune cells and led us to conclude that the net effect of IL-15 in this cancer model is promotion of tumor immunity.

\section{Results}

\section{IL-15 is Not Required for Lymphomagenesis}

The Tax oncogene in TAX-LUG mice is regulated by the granzyme $\mathrm{B}$ promoter and drives tumorigenesis and transformation of $\mathrm{CD} 16^{\mathrm{HI}}$ large granular lymphocytes. IL-15 expression is known to be elevated in these tumors [16] but the role of IL-15 and its effect on the tumor is unclear. To ascertain the function of IL-15 in Tax lymphoma, we created IL $15^{-/-}$TAX-LUG mice and observed that IL-15 is not required for tumor onset or growth. Instead, mice lacking IL-15 had a slightly accelerated rate of tumor onset (FIG. 1A) and a significantly increased mortality rate (FIG. 1B) compared to age-matched IL- $15^{+/+}$littermates. IL- $15^{+/}$ - littermates had an intermediate phenotype. In the absence of IL15 , tumors were composed of $\mathrm{CD} 16 / 32^{\mathrm{HI}}$ large granular lymphocytes and $\mathrm{CD} 16^{\mathrm{LO}}$ neutrophils (FIG. 1C,G,H), and mice developed splenomegaly and elevated lymphocyte counts in the peripheral blood (FIG. 1F) as is typically seen in TAX-LUG mice. [14] The most distinctive aspects of Tax-tumors were conserved even in the absence of IL-15 including splenomegaly, osteolytic bone lesions, a predominant admixture of tumor infiltrating Ly6G $^{+}$neutrophils (FIG. 1D), and constitutive NFאB activity within the malignant LGLs (FIG. 1E). These data demonstrate that IL-15 has a dramatic effect on survival but is not required for Tax-induced tumorigenesis such that neither the type of malignancy, nor the distinctive characteristics of the tumor model were modified by the absence of IL- 15 .

\section{IL-15 Represses Tumor Growth}

Instead of exposing the cytokine as a necessity for tumor development, the absence of IL-15 dramatically accelerated tumor growth (FIG. 2A). Luciferase expression resulting from Tax activity in the large tumors (FIG. 2B) correlated with the increased levels of Tax protein and RNA in IL- $15^{-1-}$ tumors (FIG. S1). Although IL- $15^{-1-}$ tumors were larger, no significant increase in the overall percentage of malignant cells in these tumors was observed (FIG. 1C insets). The level of Tax expression per tumor cell was increased in the absence of IL-15. Myeloperoxidase activity of tumor infiltrating leukocytes (FIG. 2C) also correlated with areas or Tax expression. IL-15-deficient mice developed larger tumors than their IL- $15^{+/+}$littermates and produced more tumors with a faster growth rate (FIG. 2D-F). Moreover, administration of soluble IL-15 was sufficient to transiently reduce the rate of tumor growth in $\mathrm{IL}-15^{-/-}$mice (FIG. 2G). The accelerated rate of tumor growth was not due to a greater percentage of proliferating cells within the tumor $\left(\mathrm{IL}-15^{+/+}=49 \%\right.$ vs. IL $-15^{-/-}=46 \%$ ) but was instead associated with a reduced percentage of tumor cells undergoing apoptosis (37\% vs. 12\%) (FIG. 2H, FIG. S2). Taken together these findings establish that IL-15 represses tumor growth in Tax lymphoma by promoting tumor cell death.

\section{IL-15 Regulates Tumor Infiltrating Cells and Tumor Associated Cytokines}

To better understand the mechanism by which IL-15 regulates tumor growth we examined the effect of IL-15 loss on gene expression within two tumor cell populations, CD16/32 ${ }^{\mathrm{HI}}$, which is highly enriched in $\mathrm{Tax}^{+}$malignant large granular lymphocytes, and CD $16 / 32^{\mathrm{LO}}$, which is a population of tumor infiltrating cells enriched in neutrophils. [17] Arrays were then performed to quantify RNA isolated from these tumor cell populations in IL$15^{+/+}$and IL-15 $5^{-/-}$tumors (FIG. 3A). The average results obtained from two independent tumors of each genotype identified transcripts that were affected by the absence of IL-15 (FIG. 3B, FIG. S3). Within the malignant CD16/32 ${ }^{\mathrm{HI}}$ population, IL- $1 \alpha$ and its receptor IL- $1 \mathrm{r} 1$, IL- $1 \alpha$-regulated cytokines ccl3 (aka: MIP1a) and cxcl3 (aka: MIP-2b,Gro-3), and IL-1 family member 9 were among the transcripts most highly elevated in the absence of IL-15. On the other hand, ccl8 (aka: MCP-2) and cxcl9 (aka: MIG) expression was dramatically repressed in the malignant population in the absence of IL-15.

Surprisingly, these data indicated that IL-15 gene expression was also elevated in the CD16/32 ${ }^{\mathrm{HI}}$ cells in IL- $15^{-1-}$ tumors. To resolve this discrepancy, a more detailed examination was conducted of the unsummarized data from each of the 26 oligonucleotide probes complementary to the $1.25 \mathrm{~kb}$ IL15 mRNA on the array (FIG. S4). In IL- $15^{-/-}$mice, a PGKNeo cassette replaced exons $3-5$ of the 8 exons of IL-15. This region corresponds to 9 of the 26 probes on the chip. Sequence complementary to the remaining 17 probes is still present in IL$15^{-1-}$ mice and, on average, these 17 remaining probes reveal a 5 -fold increase in IL-15 message even though the mRNA does not produce IL-15 protein. The low signal associated with the 9 probes complementary to the missing exons reflects the non-specific background signal of the chip.

Among the population of CD16/32 ${ }^{\mathrm{LO}}$ tumor infiltrating cells, transcripts of the neutrophil granule protein arginase 1 were markedly elevated. Tumor neutrophils in TAX-LUG mice are frequently hypersegmented with basophilic cytoplasm and ring forms are also abundant with prominent nucleoli. These characteristics were also evident in the absence of $\mathrm{IL}-15^{-/-}$ (FIG. 1 D,E,G,H). However, platelet clumping and an abundance of immature neutrophils in the peripheral blood in IL- $15^{-/-}$mice was also indicative of an impact of IL-15 loss on involved bone marrow. Reduction in gene expression in the CD16/32 ${ }^{\mathrm{LO}}$ population, not surprisingly, correlated with the reduction in IL15 dependent cell lineages. Transcripts found in NK and CD8 ${ }^{+}$ cytotoxic $\mathrm{T}$ cells were most reduced in the absence of IL-15 (FIG. 3B). Moreover, the decrease in CD8 cells in IL-15 tumors was confirmed by FACS (FIG. S5).

Finally, cytokine arrays were used to examine effects of IL-15 loss at the protein level in whole tumors (FIG. S6). Tumor 

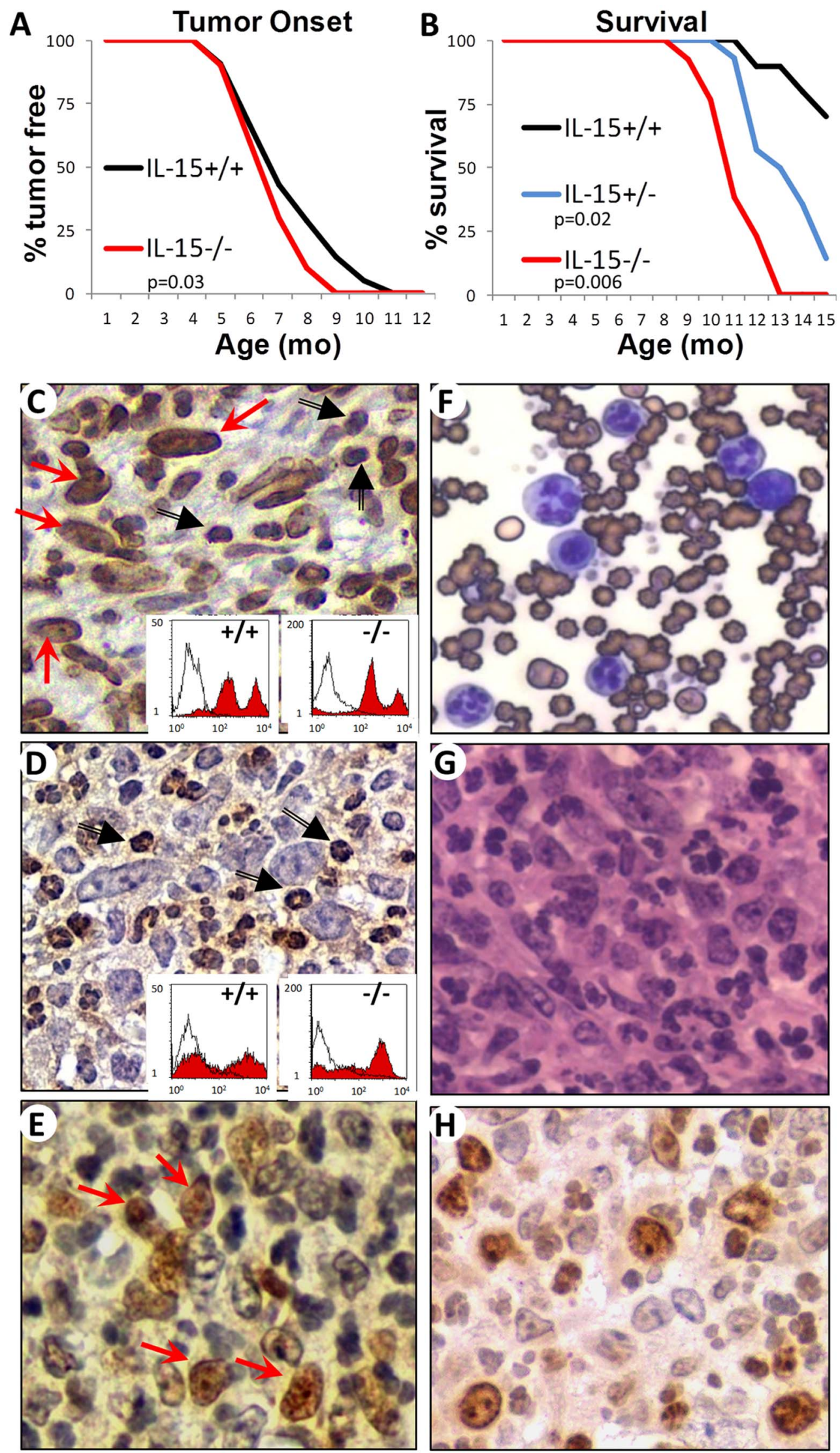

Figure 1. IL-15 is not required for Tax Tumorigenesis. A) Incidence of tumor onset in IL-15 $5^{-1-}$ TAX-LUC mice (red line, $\left.\mathrm{n}=10\right)$ compared to IL$15^{+/+}$TAX-LUC littermate control mice (black line, $n=21$ ), $p=0.03$ (2-tailed, paired Student's T Test). B) Survival curve comparing IL-15 ${ }^{-/-}$TAX-LUC 
mice (red line, $n=13$ ) and IL-15 ${ }^{+/-}$TAX-LUC mice (blue line, $n=14$ ) to IL-15 ${ }^{+/+}$TAX-LUC littermate control mice (black line, $n=10$ ). $p=0.006$ for KO vs. WT and $p=0.02$ for HET vs. WT. C) Image of CD16/32 immunohistochemistry of IL-15 $5^{-1}$ TAX-LUC tumor sections in which the malignant large granular lymphocytes (red arrows) are stained along with an admixture of tumor infiltrating neutrophils (black arrows). Insets are FACS histograms of homogenates from IL-15 $5^{+/+}$TAX-LUC or IL-15 $5^{-/-}$TAX-LUC tail tumors unstained (white curves) or stained with anti-CD16/32 FITC (red curves). D) Image of Ly6G immunohistochemistry of IL-15 ${ }^{-1-}$ TAX-LUC tumor sections in which tumor infiltrating neutrophils (black arrows) are stained. Insets

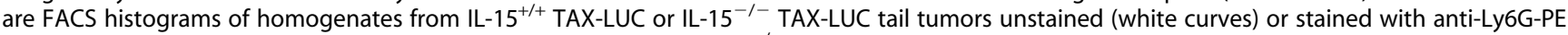
(red curves). E) Image of phosho-RelA immunohistochemistry of IL-15 ${ }^{-/-}$TAX-LUC tumor sections in which many of the malignant large granular lymphocytes (red arrows) show nuclear localization as a marker of NFKB activation. F) Image of a peripheral blood smear (Wright's stain). G) Hematoxylin and eosin stained IL-15 $15^{-1-}$ TAX-LUC tumor section. H) Image of Ki67 immunohistochemistry of IL-15 ${ }^{-1-}$ TAX-LUC tumor sections. doi:10.1371/journal.pone.0085028.g001

homogenates include epithelial, stromal, endothelial and tumor infiltrating cell types in addition to those analyzed by RNA array. The most pronounced effect of IL-15 loss on tumor-associated cytokine protein was on IL-6, which was reduced an average of 7fold in IL-15 ${ }^{-/-}$Tax tumors. Since this effect was not seen in the CD16/32 $2^{+}$populations, IL-15 may be important in regulation of IL-6 in $\mathrm{T}$ cells or other CD16/32- cells in the tumor microenvironment. It is also noteworthy that, unlike IL-1 $\beta$, the elevated levels of IL- $1 \alpha$ in normal skin keratinocytes was sufficiently high in this assay to mask any quantifiable effect of IL-15 on IL- $1 \alpha$ protein expression within the underlying tumor. Thus, proliferating in the context of significantly reduced numbers of NK and CD8 cells, the malignant population of CD16/32 ${ }^{\mathrm{HI}}$ LGLs within IL- $15^{-/}$tumors expressed elevated IL- $1 \alpha$ and IL$1 \alpha$-regulated transcripts and recruited activated tumor-infiltrating neutrophils.

\section{Loss of IL-15 Exposes IL- $1 \alpha$ as a Regulator of Tumor Growth and Potential Therapeutic Target}

Elevated levels of tumor-cell associated IL- $1 \alpha$ protein were present in formalin-fixed paraffin embedded sections of IL-15 $5^{-/-}$ tumors (Fig. 4A, Fig. S2). Interestingly, IL- $1 \alpha$ abundance in the skin surrounding the tumor was independent of IL-15. If IL- $1 \alpha$ expression in tumor cells is elevated in the absence of IL-15dependent cell-mediated immunity, and it contributes to tumor cell death in the context of a competent immune system, we asked if IL- $1 \alpha$ could be used as a therapeutic target. Two approaches were used to interrogate the potential of IL- $1 \alpha$ targeted therapies in this model. In one experiment, neutralizing anti-IL- $\alpha$ antibodies were administered to IL- $15^{+/+}$TAX-LUC mice and over the course of treatment, tumor growth was reduced compared to vehicle (FIG. 4B). Examination of anti-IL-1 $\alpha$ treated tumors upon necropsy revealed an increase in tumor infiltrating $\mathrm{CD}^{+}$and $\mathrm{CD}^{+} \mathrm{T}$ cells and a decrease in the populations of both malignant LGLs and neutrophils. The antibody-based therapy could be inhibiting IL- $1 \alpha$ signaling and/or may be targeting tumor cells for antibody dependent cell cytotoxicity (ADCG). To distinguish between these possibilities, Anakinra, a recombinant form of the IL-1 receptor antagonist, was administered to tumor bearing mice to block IL-1 signaling (FIG. 4C). In this experiment, two distinct responses were observed. Anakinra was tumoristatic in 7 of 9 tumors resulting in elevated percentages of $\mathrm{CD}^{+}$and $\mathrm{CD}^{+}$ $\mathrm{T}$ cells and fewer tumor-associated neutrophils. However, in 2 of 9 tumors, Anakinra treatment resulted in accelerated tumor growth, higher numbers of LGLs, and had no effect on $\mathrm{CD}^{+} \mathrm{T}$ cells or neutrophils. These data identify IL- $1 \alpha$ and IL-1 signaling as potential therapeutic targets.

\section{Discussion}

This study was designed to determine whether IL-15 expression is a necessary prerequisite for lymphoma development in this mouse model, and whether systemic modulation of IL-15 activity is more likely to repress or stimulate similar malignancies in vivo.
In the absence of IL-15, TAX-LUG mice developed larger, more aggressive tumors that resulted in accelerated mortality. The increase in tumor growth was transiently reversed in the presence of soluble IL-15 and the dearth of $\mathrm{NK}$ and $\mathrm{CD}^{+} \mathrm{T}$ cells in IL$15^{-/-}$mice is the likely cause of this phenotype. Interestingly, rapid tumor growth immediately followed the growth inhibitory effects of soluble IL-15 leaving open the possibility that administration of IL-15 may simultaneously promote tumor cell growth and cell mediated tumor immunity in vivo.

While IL-15 was clearly not required for tumor growth in this model, IL-15 was essential for anti-tumor immunity. Because IL15 is required for production of functional $\mathrm{NK}$ and $\mathrm{CD} 8^{+} \mathrm{T}$ cells, this model was not intended to distinguish between the effects of functional defects in $\mathrm{NK}$ and $\mathrm{CD}^{+} \mathrm{T}$ cells versus lack of recruitment. Instead, the tumor environment in IL- $15^{-/-}$mice, with reduced selective pressure of cellular cytotoxicity, revealed an unexpected transcriptional profile in the malignant cells that is apparently masked by tumor immunity. These masked targets carry potential as biomarkers capable of recruiting or activating an anti-tumor immune response. This study revealed one such target in the malignant cells to be IL- $1 \alpha$.

\section{IL- $1 \alpha$ and Tumor Immunity}

While IL- $1 \alpha$ and IL- $1 \beta$ can have similar biological activities in recombinant or secreted form, these closely related cytokines seem to play very different roles in tumor immunology. [18] IL-1 $\beta$ is active only as a secreted product but IL- $1 \alpha$ is also active in its cellassociated forms, as an intracellular precursor or in its membrane bound forms. Membrane-associated IL- $1 \alpha$ can signal in a juxtracrine manner [19] and is highly immunostimulatory, [20] capable of activating CTL and NK cells and promoting antitumor immunity and tumor regression. [21-24] In the absence of cell-mediated immunity both IL- $1 \alpha$ and IL- $1 \beta$ promote tumor growth. [25] However in an immune-competent host, IL- $1 \alpha$ appears to be a key regulator of innate immunosurveillance mechanisms that promote cell-mediated repression of malignancy. $[24,26]$ IL- $1 \beta$, on the other hand, leads to immune suppression of the host by activation and recruitment of $\mathrm{T}_{\text {reg }}$ cells and $\mathrm{CD}_{1} 1 \mathrm{~b}^{+} /$ $\mathrm{Grl}^{+}$immature myeloid cells and it promotes systemic inflammation, tumor invasiveness and tumor angiogenesis. [21,22,27].

Intracellular IL- $1 \alpha$ carries a nuclear localization signal in its $\mathrm{N}$ terminus and is a chromatin associated cytokine [28] that can regulate gene expression by binding the p300-PCAF complex and activating genes in the $\mathrm{NF \kappa B}$ and AP-1 pathways. [29,30] Intracellular IL- $1 \alpha$ can also be released during cell necrosis, where its receptor-interacting C-terminus is capable of recruiting infiltrating myeloid cells. [28] Under hypoxic conditions, HIF-1 $\alpha$ regulates IL-1-dependent recruitment of myeloid cells [31] such that IL- $1 \alpha$ expression correlates with the infiltration of neutrophils, whereas IL- $1 \beta$ is associated with the recruitment of macrophages. $[28,32]$ In sum, IL- $1 \alpha$ and IL- $1 \beta$ are unique therapeutic targets and cell-associated IL- $1 \alpha$ is well-suited to serve as a tumor biomarker, CTL adjuvant, and target for antibody or complement-mediated cellular cytotoxicity. 

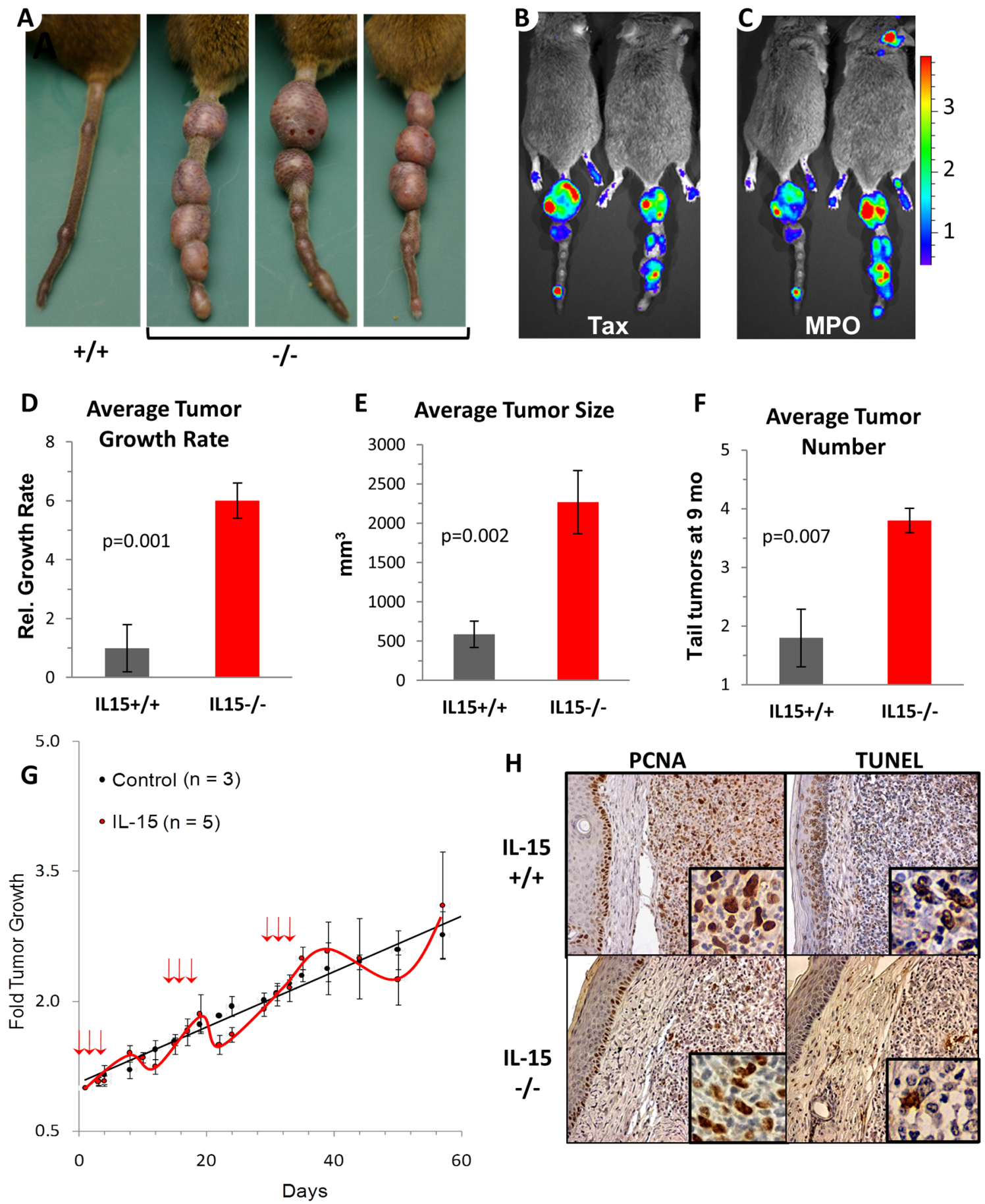

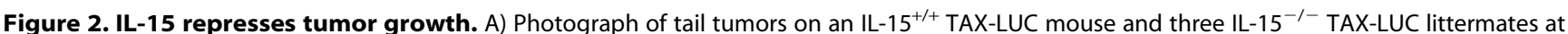
8 mo of age. B,C) Bioluminescent imaging of IL-15 $15^{-/}$TAX-LUC mice to detect Tax activity (using D-luciferin to measure LTR-Luciferase activity), and neutrophil infiltration (using Luminol to measure myeloperoxidase released from degranulating, tumor-infiltrating neutrophils). D-F) Bar graphs representing the average tumor growth rate, the average tumor size, and the average number of tail tumors per mouse on IL-15 $5^{+/+}(\mathrm{n}=5)$, IL-15 $5^{-/-}$ $(n=10)$ TAX-LUC mice. Weekly, bi-directional caliper measurements were taken for each tumor from tumor onset to death. Tumor volume doubling time was calculated for each tumor. To determine the growth rate, the average doubling time for IL-15 $5^{++}$TAX-LUC mice was set to 1 to obtain the relative average doubling time of IL-15 $15^{-1-}$ TAX-LUC mice. Tumor size is shown as tumor volume in $\mathrm{mm}^{3}$. Number of tail tumors was calculated at 9 months of age. Error bars indicate the standard deviation and $\mathrm{p}$ values show 2-tailed, paired Student's T Tests. G) The effect of murine IL-15 on tumor growth. IL-15 $5^{-1-}$ TAX-LUC mice received saline (black curve, $\mathrm{n}=3$ ) or mIL-15 (red curve, $\mathrm{n}=5$ ) at times indicated by red arrows. Error bars represent standard deviation of average tumor volume as measured by calipers. H) Images of representative immunohistochemistry stains for proliferation (proliferating cell nuclear antigen; PCNA) and apoptosis (TUNEL) on IL-15 ${ }^{+/+}$TAX-LUC and IL-15 ${ }^{-/-}$TAX-LUC tail tumor sections. doi:10.1371/journal.pone.0085028.g002 


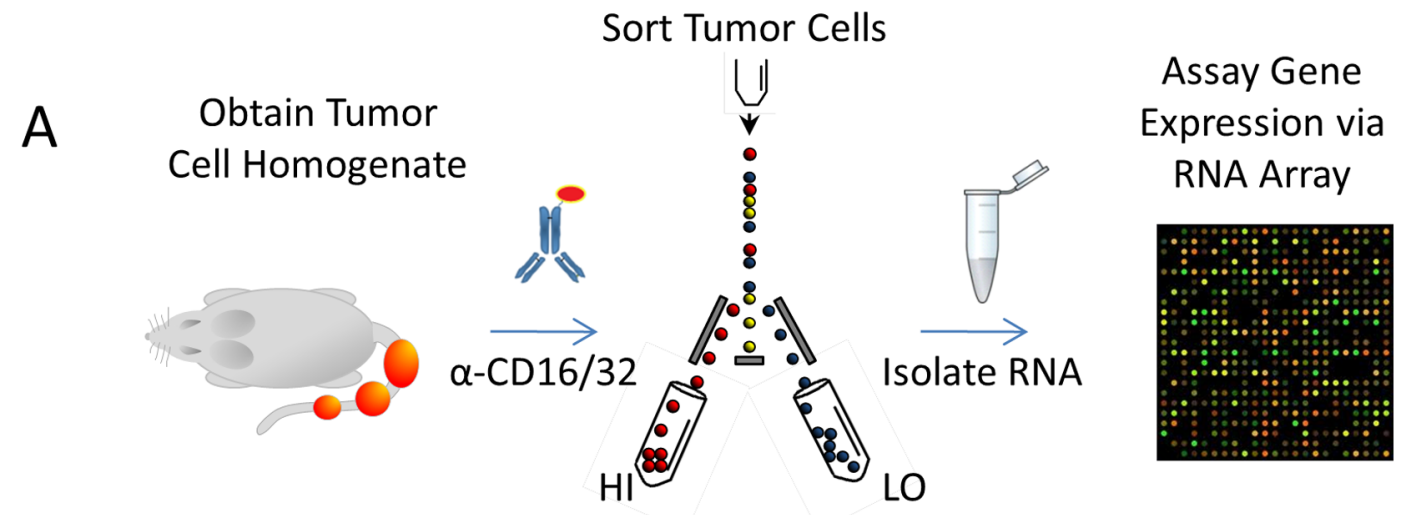

B

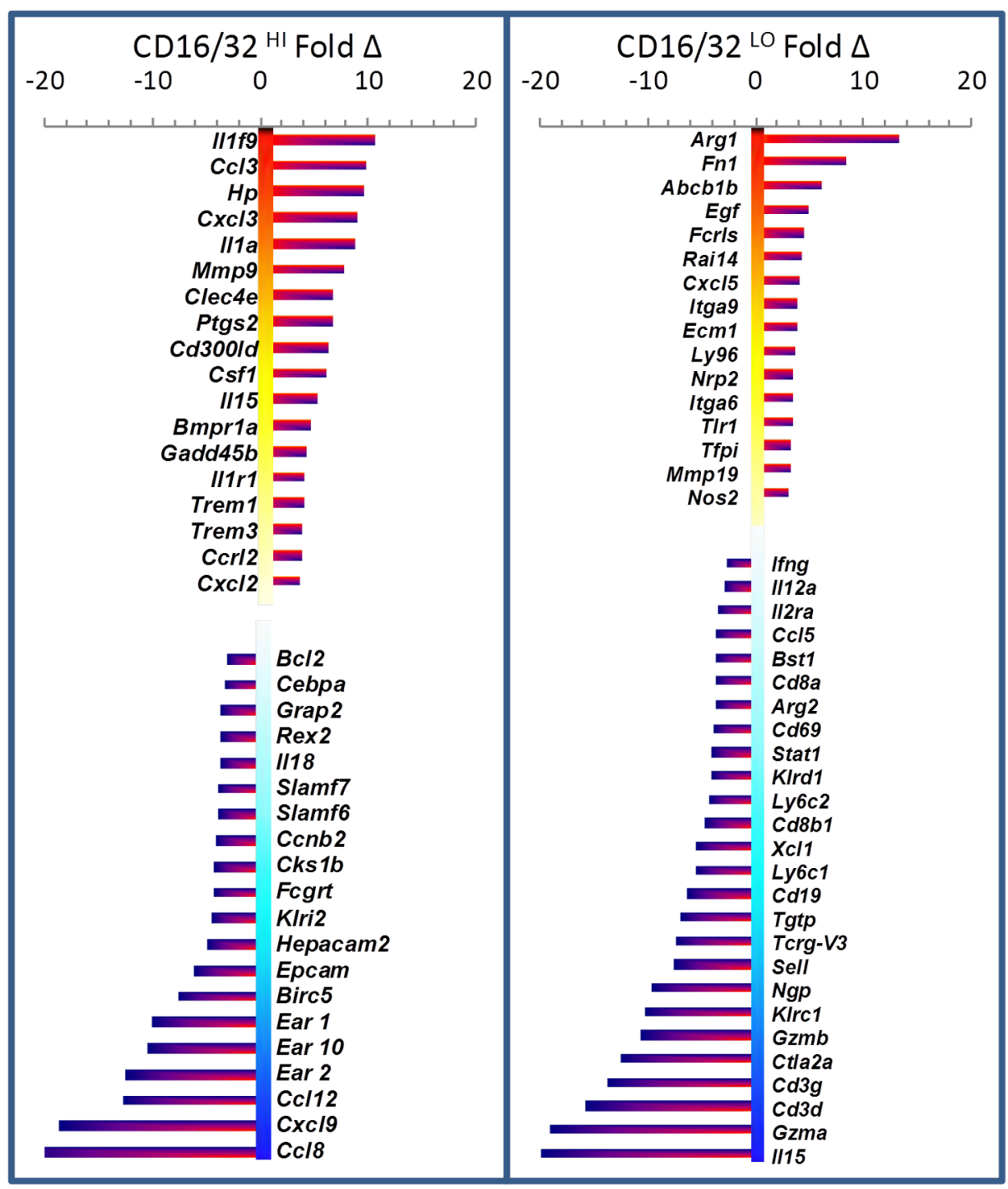

Figure 3. IL-15 regulates tumor infiltrating cells and tumor associated cytokines. A) Schematic representation of experimental design. RNA

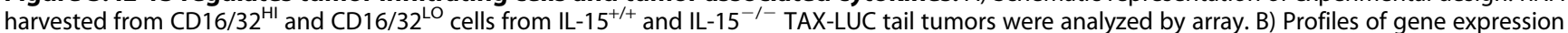
changes associated with the absence of IL-15 in $\mathrm{CD} 16 / 32^{\mathrm{HI}}$ and $\mathrm{CD} 16 / 32^{\mathrm{LO}}$ tumor cell populations.

doi:10.1371/journal.pone.0085028.g003 
A

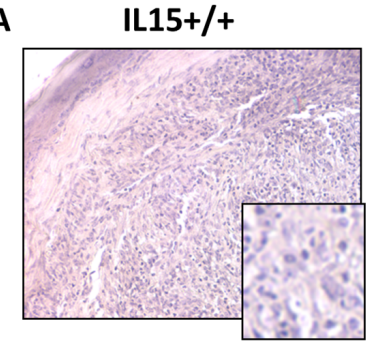

IL15+/+

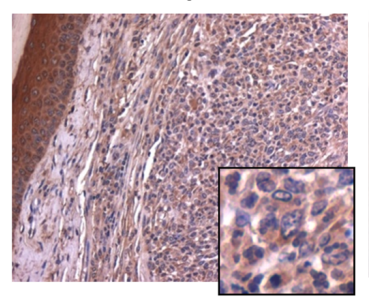

IL15-/-

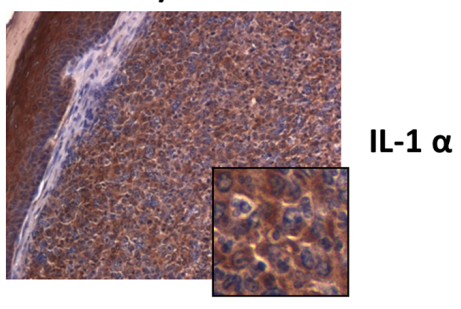

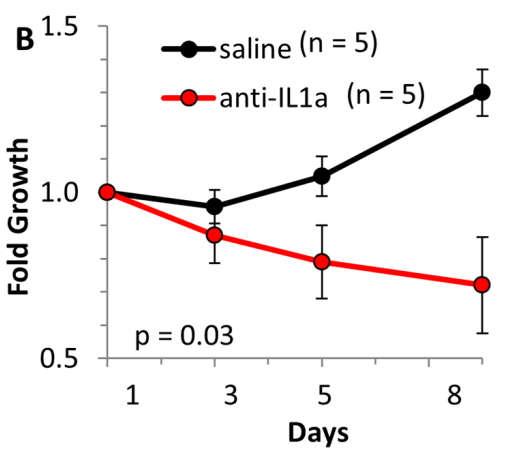

C

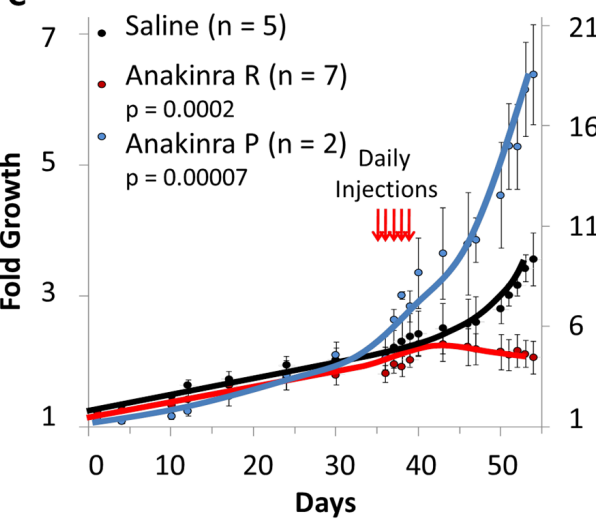

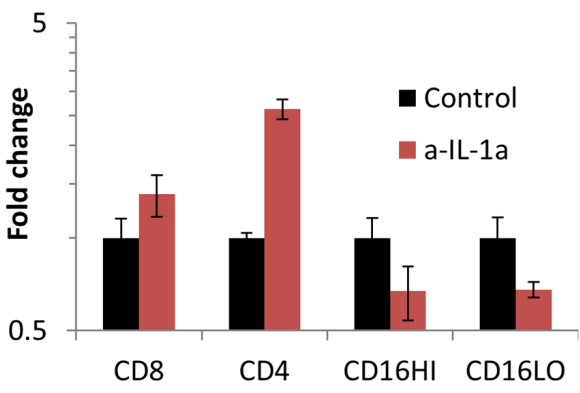

21

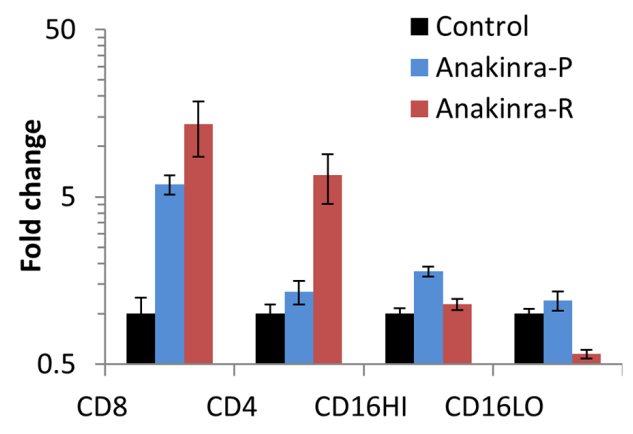

Figure 4. Loss of IL-15 exposes IL-1 $\alpha$ as a regulator of tumor growth and potential therapeutic target. $\mathrm{A}) \mathrm{Images}$ of IL-1 $\alpha \mathrm{IHC}$ in IL-15 $5^{+/+}$ (middle) and IL-15 $5^{-/-}$(right) TAX-LUC tumor sections compared to control lacking primary IL-1 $\alpha$ antibody (left). B) The effect of anti-IL-1 $\alpha$ antibodies (red curve, $\mathrm{n}=5$ ) on tumor growth compared to saline (black curve, $\mathrm{n}=5$ ) in IL-15 $5^{++} \mathrm{TAX}$-LUC mice over the course of 1 week. Error bars represent standard deviation of tumor growth, and $\mathrm{p}$ values represent 2-tailed, paired Student's T Tests. The bar graph (right) represents FACS data obtained from anti-IL-1a treated tumors (red bars) or control tumors (black bars) at necropsy showing the abundance of CD8 ${ }^{+}, \mathrm{CD} 4^{+}, \mathrm{CD} 16 / 32^{\mathrm{HI}}$, and $\mathrm{CD} 16 /$ $32^{\mathrm{LO}}$ cells present in the tumor at the experimental endpoint. Y axis is log scale and error bars represent standard deviation. C) The effect of Anakinra on tumor growth compared to saline. In 7 of 9 mice Anakinra resulted in repression of tumor growth (Anakinra R, red line), while in 2 of 9 mice Anakinra resulted in promotion of tumor growth (Anakinra $\mathrm{P}$, blue line). Graph is double- $Y$ to accommodate the scale differences between Anakinra $\mathrm{R}$ and control (left scale) versus Anakinra P (right scale). Red arrows indicate the time points of daily Anakinra injections, error bars represent standard deviations and $\mathrm{p}$ values represent 2-tailed, paired Student's T Tests. The bar graph (right) represents FACS data obtained from Anakinra treated tumors or control tumors at necropsy showing the abundance of $\mathrm{CD}^{+}, \mathrm{CD} 4^{+}, \mathrm{CD} 16 / 32^{\mathrm{HI}}$, and CD16/32 $2^{\mathrm{LO}}$ cells present in the tumor at the experimental endpoint. $Y$ axis is log scale and error bars represent standard deviation.

doi:10.1371/journal.pone.0085028.g004

\section{IL-1 $\alpha$ and HTLV Tax}

Two striking characteristics of ATLL are the long period of latency that usually precedes disease onset and the low penetrance of ATL among infected carriers. Among the mechanisms thought to underlie these characteristics, one major factor is the role of CTL and host immunity. [33,34] It has been established that the Tax oncogene is an antigenic target of the adaptive immune response in HTLV-1 infected individuals. [35] Tax is also known to activate the IL- $1 \alpha$ promoter, [36] several mouse models have demonstrated elevated levels of IL- $1 \alpha$ in response to Tax expression, $[13,17,37]$ and HTLV-1+ T cell clones produce high levels of IL- $1 \alpha$ in culture. [38] Interestingly, Tax expression is largely restricted or absent in primary ATL cells $[39,40]$ and the same is true for IL- $1 \alpha$. [41] Compared to IL- $1 \beta$, IL- $1 \alpha$ is also less abundant in primary tissue samples of other human malignancies including melanoma, colon carcinoma, and non-small cell lung carcinoma. [42] These data are consistent with the hypothesis that there is a selection against IL- $1 \alpha$ expression in ATL cells, and other malignancies, in vivo. Our data provides a mechanistic explanation for these observations in ATL. Tax activity leads to IL- $1 \alpha$ expression which recruits and activates cell-mediated killing of IL- $1 \alpha$-expressing, HTLV-1 infected cells. This is corroborated by the fact that depletion of IL- $1 \alpha$ in a Tax transgenic mouse model reduced Tax-induced autoimmunity. [43] What remains to 
be determined is whether IL- $1 \alpha$-directed therapies can enhance killing of HTLV infected cells in vivo, reduce viral load, or target Tax-dependent mechanisms of ATL progression.

\section{Translational Implications of IL-15 and IL-1 $\alpha$}

IL-15 is already recognized as a promising candidate for tumor immunotherapy. [1,2] The principle findings in this study are that IL-15 is critical in the inhibition of tumor growth in this model and that IL- $1 \alpha$ expression is targeted by IL-15-dependent anti-tumor activity (FIG. 5). Taken together these findings provide a rationale for testing IL-15, an anti-IL- $1 \alpha$ antibody or a combination of both in lymphoma. The combination of IL-15 and cetuximab increased ADCG activity against triple negative breast cancer cell lines and enhanced cetuximab efficacy against HER1-positive head and neck cancer. [44,45] IL-15 has also been shown to enhance rituximab-dependent cytotoxicity against chronic lymphocytic leukemia cells. [46] Moreover, the combination of IL-15 and anti-CD40 is more efficacious than either therapy alone in mouse models of metastatic colon cancer or metastatic renal cell carcinoma. [47-49] An anti- IL- $\alpha$ monoclonal antibody (MABpl, Xbiotech) is currently undergoing clinical trials for treatment of advanced leukemia and other cancers, and has shown evidence of anti-tumor activity. [50] Alone and in combination, IL-15 and anti-IL-1 $\alpha$ therapies are worth exploring against hematopoietic malignancies.

\section{Materials and Methods}

\section{Animals}

IL-15 ${ }^{-1-}$ mice on a C57BL/6 background (3, Taconic) were intercrossed with TAX-LUC transgenic mice on a C57BL/ $6 \times$ FVB background. [14] The use of murine models and tissues in

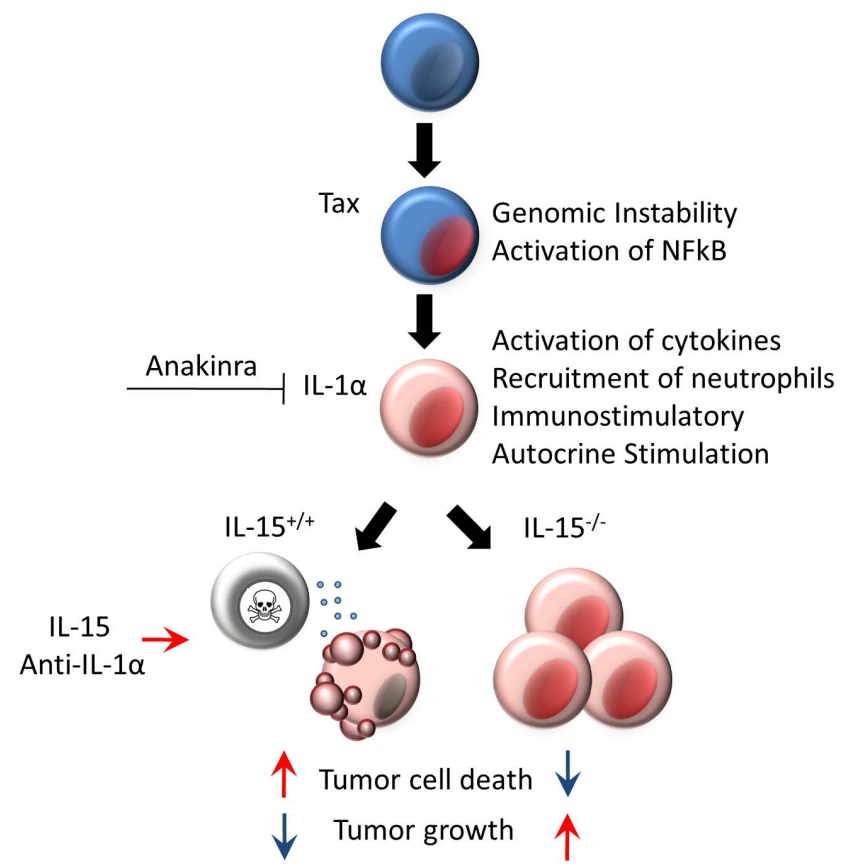

Figure 5. Model. A schematic representation of the principle conclusions; IL-15 is not required for tumor growth but is required for tumor immunity, $\mathrm{IL}-1 \alpha$ is more abundant and tumor growth is accelerated in the absence of $\mathrm{IL}-15$, and therapies that target IL-1 and/or promote cell-mediated tumor immunity should be explored in lymphoma or other malignancies.

doi:10.1371/journal.pone.0085028.g005 this study was carried out in strict accordance with the recommendations in the Guide for the Care and Use of Laboratory Animals of the National Institutes of Health. Mice were housed under pathogen-free conditions according to the guidelines of the Division of Comparative Medicine and all experiments were approved by the Animal Studies Committee, Washington University School of Medicine under ASG protocol \#20100026.

\section{Histology and Immunohistochemistry}

Tumor or organ tissue was fixed in $10 \%$ buffered formalin and paraffin-embedded sections were processed for immunohistochemical stains. Slides were heated to $55^{\circ} \mathrm{C}$ for 10 minutes, deparaffinized in xylene, and rehydrated in deionized water. For antigen retrieval/unmasking, slides were boiled (under pressure) in citric acid buffer ( $\mathrm{pH}$ 6) for 15 minutes and allowed to cool to room temperature. In some cases, slides were also treated with proteinase $\mathrm{K}(20 \mathrm{ug} / \mathrm{mL}$ in $\mathrm{TE}, \mathrm{pH} 8)$ for 2 minutes at room temperature, and washed with PBS. Endogenous peroxidase activity was blocked by incubating the slides in $0.3 \%$ hydrogen peroxide solution for 5 minutes. For Ki67 staining, slides were then blocked in normal equine serum (Jackson ImmunoResearch Laboratories Inc.) for 15 minutes and rinsed. Slides were soaked in rabbit polyclonal anti-Ki67 (Vector Laboratories) overnight at $4^{\circ} \mathrm{C}$. Slides were then washed and incubated in ImmPRESS reagent anti-rabbit IgG (Vector Laboratories) for 30 minutes at room temperature, incubated in DAB peroxidase substrate, rinsed, and coverslipped. TUNEL (terminal deoxynucleotidyl transferase dUTP nick end labeling) staining was conducted according to manufacturer's protocol (Trevigen). For CD16/32 staining additional blocking steps with avidin D solution (Vector Laboratories) and for $15 \mathrm{~min}$ at room temp, followed by biotin for 15 minutes at room temperature was included prior to incubation in primary biotin rat anti-mouse CD16/32 antibody (BD Pharmingen) overnight at $4^{\circ} \mathrm{C}$. Rabbit polyclonal anti-PCNA (abcam), rabbit monoclonal anti-phosphoRel-A (Cell Signaling Technology Inc), rat anti-mouse Ly6G (eBioscience), and goat polyclonal anti-rat IL- $1 \alpha$ (Santa Cruz biotechnology, Inc) primary antibodies were used. Negative controls lacking primary antibody were included for each stain. Sections were visualized with a Nikon Eclipse E400 microscope and digital images were obtained using a Magnafire camera and software (Optronics).

\section{Treatment Regimens}

Soluble mIL-15: IL-15 ${ }^{-/-}$TAX-LUG mice received subcutaneous injections of saline or $100 \mathrm{ng}$ of recombinant mouse IL15 (MACS Miltenyi Biotech), 3 times per week every other week for 5 weeks. Bi-directional caliper measurements of tumors were taken three times per week for 2 months. Anti-IL- $1 \alpha$ antibody: IL$15^{+/+}$TAX-LUC mice received injections of 50 ug LEAFpurified, anti-mouse IL- $1 \alpha$ antibody (BioLegend) or saline i.p. every other day for one week and tumors were measured with calipers. Anakinra: IL- $15^{+/+}$TAX-LUG received daily i.p. injections of $30 \mathrm{mg}$ Anakinra (Swedish Orphan Biovitrum AB) or saline. Tumors were measured for 35 days prior to treatment and 15 days after treatment with calipers.

\section{Bioluminescent Imaging}

The IVIS100 system (Xenogen) was used to image bioluminescence in anesthetized mice (isoflurane inhalation). Standard imaging parameters included D-luciferin dose $50 \mathrm{mg}$ i.p; luminol dose $200 \mathrm{mg} / \mathrm{kg}$ i.p; exposure $300 \mathrm{sec}$; binning 4; f/stop 1; no optical filter. Color scale unless otherwise indicated is $\times 10^{4}$ photons $/ \mathrm{sec} / \mathrm{cm}^{2} / \mathrm{sr}$. 


\section{Flow Cytometry}

Cell suspensions derived from organs or tumor homogenates from IL- $15^{-/-}$or IL- $15^{+/+}$mice were blocked with mouse BD FC Block (Rat anti-mouse CD16/32; BD Pharmingen) for 10 minutes and stained with anti-CD16/32-FITC, anti-CD4-APC, anti-CD8$\mathrm{PE}$, and anti-Ly6G-PE antibodies (eBioscience) for 30 minutes at $4^{\circ} \mathrm{C}$, washed, filtered and analyzed on a BD FACSCalibur (Becton Dickinson).

\section{RNA Array}

Tail tumors were excised from two IL- $15^{-/-}$TAX-LUC mice and two IL- $15^{+/+}$TAX-LUG mice. Single cell suspensions were made, counted, re-suspended in PBS+1\%BSA, blocked with $\mathrm{FC}_{\mathrm{C}}$ block, stained with anti-CD16/32 for $30 \mathrm{~min}$ at $4^{\circ} \mathrm{C}$, and sorted using Sony iCyt Reflection Cell Sorter. Total RNA was harvested from $\mathrm{CD} 16^{\mathrm{HI}}$ and $\mathrm{CD} 16^{\mathrm{LO}}$ tumor cell populations using RNAeasy (Qiagen), quantified by spectrophotometry, and submitted for array analysis (Affymetrix Gene Chip, Whole Transcript 1.0ST). Averaged signal intensities for each probe set were analyzed (Partek) comparing CD16/32 ${ }^{\mathrm{HI}}$ from each genotype and CD 16/32 ${ }^{\mathrm{LO}}$ sets from each genotype by 1-way ANOVA and then filtered for expression changes $>3$-fold. Original data files are available (GEO accession \# GSE46072).

\section{Protein Array}

Tail tumors and normal tail tissue from IL- $15^{-/-}$and IL- $15^{+/+}$ TAX-LUC mice were excised and homogenized in PBS with protease inhibitors $(10 \mu \mathrm{g} / \mathrm{mL}$ Aprotinin, $10 \mu \mathrm{g} / \mathrm{mL}$ Leupeptin, and $10 \mu \mathrm{g} / \mathrm{mL}$ Pepstatin) and Triton X-100 was added to a final concentration of $1 \%$. Samples were then frozen at $-80^{\circ} \mathrm{C}$, thawed, centrifuged at $10,000 \times \mathrm{g}$ for 5 minutes to remove cellular debris, quantified, and analyzed using a Proteome Profiler Mouse Cytokine Antibody Array (R\&D Systems) according to manufacturer's instructions.

\section{RT-PCR}

$2 \mu \mathrm{g}$ of RNA obtained from sorted cells was subjected to DNase I digestion (Invitrogen) and RT-PCR was carried out using SuperScript III First-Strand Synthesis system (Invitrogen) according to manufacturer's instructions. $100 \mathrm{ng}$ of cDNA template was used for each reaction. Qualitative RT-PCR was performed using a two-phase, step-down PCR method; 16 cycles of $94^{\circ} \mathrm{C}$ for $30 \mathrm{~s}$, $64^{\circ} \mathrm{C}\left(-0.5^{\circ} \mathrm{C} /\right.$ cycle $)$ for $30 \mathrm{~s}, 72^{\circ} \mathrm{C}$ for $30 \mathrm{~s}$, followed by 10 cycles of $94^{\circ} \mathrm{C}$ for $30 \mathrm{~s}, 56^{\circ} \mathrm{C}$ for $30 \mathrm{~s}, 72^{\circ} \mathrm{C}$ for 30 .

\section{Western Blot}

Tumor cells harvested from IL- $15^{-/-}$and IL- $15^{+/+}$TAX-LUC mice were centrifuged, rinsed, resuspended in RIPA buffer, sonicated, boiled in SDS buffer for $15 \mathrm{~min}$, and run on $15 \%$ SDS-PAGE at $4^{\circ} \mathrm{C}$. Blots were probed using a rabbit anti-Tax primary antibody.

\section{Supporting Information}

Figure S1 Tax Expression. RNA and protein obtained from tumor cells harvested from IL- $15^{-/-}$and IL- $15^{+/+}$TAX-LUG mice was used to detect Tax RNA by RT-PCR and Tax protein by Western Blot normalized against GAPDH and actin loading controls respectively.

(TIF)

Figure S2 TUNEL, PGNA, and IL-1 $\alpha$ IHG in IL-15 ${ }^{+/+}$and IL-15 $^{- \text {/ }}$ tumors. Images of representative immunohistochem- istry (IHC) stains for apoptosis (Terminal deoxynucleotidyl transferase dUTP nick end labeling; TUNEL), proliferation (proliferating cell nuclear antigen; PGNA) and interleukin-1 alpha (IL- $1 \alpha$ ) on IL- $15^{+/+}$TAX-LUG (top row) and IL- $15^{-/-}$TAXLUC (bottom row) tail tumor sections.

(TIF)

Figure S3 RT-PGR confirmation of selected differentially expressed mRNAs. RNA was obtained from CD16/ $32^{\mathrm{HI}}$ and CD 16/32 ${ }^{\mathrm{LO}}$ sorted tumor cells harvested from IL-15 ${ }^{-/-}$ and IL- $15^{+/+}$TAX-LUG mice.

(TIF)

Figure S4 IL-15 mRNA is elevated in the malignant CD16/32 ${ }^{\mathrm{HI}}$ cells in $\mathrm{IL-15}^{-/-}$Tax tumors. A) Raw ("unsummarized") data from each of the 26 oligonucleotide probes complementary to the $1.25 \mathrm{~kb}$ IL-15 mRNA on the array. Red bars and black bars represent data obtained from CD 16/32 $\mathrm{HI}$ cells from tail tumors arising in IL- $15^{-/-}$and IL- $15^{+/+}$TAXLUC mice respectively. B) Alignment showing the locations of each of the 26 oligonucleotide probes relative to the exon locations in the mRNA. In IL-15 $5^{-/-}$mice the PGK Neo cassette replaces exons 3-5 of the IL-15 mRNA which corresponds to probes 7-15 in the array. Probes $7-15$ in the IL- $15^{-/-}$tumors constitute the background of the array and average a 1.54 fold decrease vs. IL$15^{+/+}$compared to the 5.26 fold increase in the average of the remaining probes.

(TIF)

Figure S5 Tumor infiltrating $\mathrm{CD8}^{+}$cells are reduced in IL-15 $^{- \text {/- }}$ Tax tumors. Dot plot histograms of $\mathrm{CD}^{+}$(Y-axis) and $\mathrm{CD}^{+}(\mathrm{X}$-axis) cells present in tumor homogenates from IL$15^{+/+}$and IL- $15^{-1-}$ Tax tumors.

(TIF)

Figure S6 Gytokines elevated in Tax Tumors in the presence and absence of IL-15. Lysates were obtained from normal tail tissue and whole tail tumors from IL- $15^{-/-}$and IL$15^{+/+}$mice and analyzed using the mouse Proteome Profiler. Representative images are shown from one array next to densitometry analysis averaging the results of $\mathrm{n}=5 \mathrm{IL}-15^{-/-}$ arrays. Densitometry value in normal IL- $15^{-1-}$ tail tissue is shown and set to 1. A 7-fold decrease in IL-6 protein was consistently detected in IL- $15^{-/-}$tumors compared to IL- $15^{+/+}$tumors.

(TIF)

\section{Acknowledgments}

We thank the Alvin J. Siteman Cancer Center at Washington University School of Medicine and Barnes-Jewish Hospital in St. Louis, for the use of the Siteman Flow Cytometry Core, which provided the cell sorting service, and for use of the Molecular Genomic Analysis (MGA) Core in the Laboratory for Clinical Genomics, which provided Affymetrix array analysis and support. We thank David Piwnica-Worms and the Molecular Imaging Center at the Bright Institute which provided access to the IVIS 100 system and imaging reagents. We thank Stefan Niewiesk and the Center of Retrovirus Research at the Ohio State University College of Veterinary Medicine for providing tissue pathology support. We also thank Shibani Mitra-Kaushik, Sirosh Bokhari, and Matthew Cherian for excellent advice, performing assays, and/or providing technical assistance.

\section{Author Contributions}

Conceived and designed the experiments: DAR JCH LR. Performed the experiments: DAR JCH. Analyzed the data: DAR JCH LR. Wrote the paper: DAR. Edited the manuscript: DAR JCH LR. 


\section{References}

1. Gillgrass A, Ashkar A (2011) Stimulating natural killer cells to protect against cancer: recent developments. Expert Rev Clin Immunol 7: 367-82.

2. Jakobisiak M, Golab J, Lasek W (2011) Interleukin 15 as a promising candidate for tumor immunotherapy. Cytokine Growth Factor Rev 22: 99-108.

3. Kennedy MK, Glaccum M, Brown SN, Butz EA, Viney JL, et al. (2000) Reversible defects in natural killer and memory CD8 $\mathrm{T}$ cell lineages in interleukin 15-deficient mice. J Exp Med 191: 771-80.

4. Burton JD, Bamford RN, Peters C, Grant AJ, Kurys G, et al. (1994) A lymphokine, provisionally designated interleukin $\mathrm{T}$ and produced by a human adult T-cell leukemia line, stimulates T-cell proliferation and the induction of lymphokine-activated killer cells. Proc Natl Acad Sci U S A 91: 4935-9.

5. Bamford R, Battiata A, Burton J, Sharma H, Waldmann T (1996) Interleukin (IL) 15/IL-T production by the adult T-cell leukemia cell line HuT-102 is associated with a human T-cell lymphotrophic virus type I region/IL-15 fusion message that lacks many upstream AUGs that normally attenuates IL-15 mRNA translation. Proc Natl Acad Sci U S A 93: 2897-902.

6. Hodge DL, Yang J, Buschman MD, Schaughency PM, Dang H, et al. (2009) Interleukin-15 enhances proteasomal degradation of Bid in normal lymphocytes. Implications for large granular lymphocyte leukemias. Cancer Res 69: 39863994

7. Shah MV, Zhang R, Irby R, Kothapalli R, Liu X, et al. (2008) Molecular profiling of LGL leukemia reveals role of sphingolipid signaling in survival of cytotoxic lymphocytes. Blood 112: 770-781.

8. Kuniyasu H, Ohmori H, Sasaki T, Sasahira T, Yoshida K, et al. (2003) Production of interleukin 15 by human colon cancer cells is associated with induction of mucosal hyperplasia, angiogenesis, and metastasis. Clin Cancer Res 9: $4802-4810$.

9. Trentin L, Cerutti A, Zambello R, Sancretta R, Tassinari C, et al. (1996) Interleukin-15 promotes the growth of leukemic cells of patients with B-cell chronic lymphoproliferative disorders. Blood 87: 3327-3335.

10. Sato N, Sabzevari H, Fu S, Petrus MN, Bamford RN, et al. (2011) Development of an IL-15-autocrine CD8 T-cell leukemia in IL-15-transgenic mice requires the cis expression of IL-15R $\alpha$. Blood 117: 4032-40.

11. Mishra A, Liu S, Sams GH, Curphey DP, Santhanam R, et al. (2012) Aberrant overexpression of IL-15 initiates large granular lymphocyte leukemia through chromosomal instability and DNA hypermethylation. Cancer Cell 22: 645-55.

12. Azimi N, Brown K, Bamford RN, Tagaya Y, Siebenlist U, Waldmann TA (1998) Human T cell lymphotropic virus type I Tax protein trans-activates interleukin 15 gene transcription through an NF-kappaB site. Proc Natl Acad Sci U S A 95: 2452-7.

13. Grossman WJ, Kimata JT, Wong FH, Zutter M, Ley TJ, Ratner L (1995) Development of leukemia in mice transgenic for the tax gene of human T-cell leukemia virus type I. Proc Natl Acad Sci U S A 92: 1057-61.

14. Rauch D, Gross S, Harding J, Niewiesk S, Lairmore M, et al. (2009) Imaging spontaneous tumorigenesis: inflammation precedes development of peripheral NK tumors. Blood 113: 1493-500.

15. Rauch D, Gross S, Harding J, Bokhari S, Niewiesk S, et al. (2009) T cell activation promotes tumorigenesis in inflammation-associated cancer. Retrovirology 6: 116 .

16. Portis T, Harding J, Ratner L (2001) The contribution of NF-kappa B activity to spontaneous proliferation and resistance to apoptosis in human T-cell leukemia virus type 1 Tax-induced tumors. Blood 98: 1200-8.

17. Gao L, Deng H, Zhao H, Hirbe A, Harding J, et al. (2005) HTLV-1 Tax transgenic mice develop spontaneous osteolytic bone metastases prevented by osteoclast inhibition. Blood 106: 4294-302.

18. Dinarello CA (2009) Immunological and inflammatory functions of the interleukin-1 family. Annu Rev Immunol 27: 519-50.

19. Dvorkin T, Song X, Argov S, White RM, Zoller M, et al. (2006) Immune phenomena involved in the in vivo regression of fibrosarcoma cells expressing cell-associated IL-1 $\alpha$. J Leukoc Biol 80: 96-106.

20. Ben-Sasson SZ, Caucheteux S, Crank M, Hu-Li J, Paul W (2011) IL-1 acts on T cells to enhance the magnitude of in vivo immune responses. Cytokine 56: 122-

21. Song X, Voronov E, Dvorkin T, Fima E, Cagnano D, et al. (2003) Differential effects of IL-1 alpha and IL-1 $\beta$ on tumorigenicity patterns and invasiveness. J Immunol 171: 6448-56.

22. Apte RN, Dotan S, Elkabets M, White MR, Reich E, et al. (2006) The involvement of IL-1 in tumorigenesis, tumor invasiveness, metastasis and tumorhost interactions. Cancer Metastasis Rev 25: 387-408.

23. Apte RN, Dvorkin T, Song X, Fima E, Krelin Y, et al. (2000) Opposing effects of IL-1 alpha and IL-1 $\beta$ on malignancy patterns. Tumor cell-associated IL-1 alpha potentiates anti-tumor immune responses and tumor regression, whereas IL-1 $\beta$ potentiates invasiveness. Adv Exp Med Biol 479: 277-88.

24. Elkabets M, Krelin Y, Dotan S, Cerwenka A, Porgador A, et al. (2009) Hostderived interleukin-1alpha is important in determining the immunogenicity of 3methylcholanthrene tumor cells. J Immunol 182: 4874-81.

25. Nazarenko I, Marhaba R, Reich E, Voronov E, Vitacolonna M, et al. (2008) Tumorigenicity of IL-1alpha- and IL-1 beta-deficient fibrosarcoma cells. Neoplasia 10: 549-62.
26. Marhaba R, Nazarenko I, Knöfler D, Reich E, Voronov E, et al, (2008) Opposing effects of fibrosarcoma cell-derived IL-1 alpha and IL-1 beta on immune response induction. Int J Cancer 123: 134-45.

27. Song X, Krelin Y, Dvorkin T, Bjorkdahl O, Segal S, et al. (2005) CD1 lb+/Gr$1+$ immature myeloid cells mediate suppression of $\mathrm{T}$ cells in mice bearing tumors of IL-1 beta-secreting cells. J Immunol 175: 8200-8.

28. Cohen I, Rider P, Carmi Y, Braiman A, Dotan S, et al. (2010) Differential release of chromatin-bound IL- $1 \alpha$ discriminates between necrotic and apoptotic cell death by the ability to induce sterile inflammation. Proc Natl Acad Sci U S A 107: 2574-9.

29. Buryskova M, Pospisek M, Grothey A, Simmet T, Burysek L (2004) Intracellular interleukin-1alpha functionally interacts with histone acetyltransferase complexes. J Biol Chem 279: 4017-26.

30. Werman A, Werman-Venkert R, White R, Lee JK, Werman B, et al. (2004) The precursor form of $\mathrm{IL}-1 \alpha$ is an intracrine proinflammatory activator of transcription. Proc Natl Acad Sci U S A 101: 2434-9.

31. Rider P, Kaplanov I, Romzova M, Bernardis L, Braiman A, et al. (2012) The transcription of the alarmin cytokine interleukin- 1 alpha is controlled by hypoxia inducible factors 1 and 2 alpha in hypoxic cells. Front Immunol 3: 290.

32. Rider P, Carmi Y, Guttman O, Braiman A, Cohen I, et al. (2011) IL-1 $\alpha$ and IL$1 \beta$ recruit different myeloid cells and promote different stages of sterile inflammation. J Immunol 187: 4835-43.

33. Goon PK, Biancardi A, Fast N, Igakura T, Hanon E, et al. (2004) Human T cell lymphotropic virus (HTLV) type-1-specific CD8+ $\mathrm{T}$ cells: frequency and immunodominance hierarchy. J Infect Dis 189: 2294-8.

34. Rowan AG, Bangham CR (2012) Is There a Role for HTLV-1-Specific CTL in Adult T-Cell Leukemia/Lymphoma? Leuk Res Treatment 2012: 391953. doi: 10.1155/2012/391953.

35. Pique C, Connan F, Levilain JP, Choppin J, Dokhélar MC (1996) Among all human T-cell leukemia virus type 1 proteins, tax, polymerase, and envelope proteins are predicted as preferential targets for the HLA-A2-restricted cytotoxic T-cell response. J Virol 70: 4919-26.

36. Mori N, Prager D (1996) Transactivation of the interleukin-lalpha promoter by human T-cell leukemia virus type I and type II Tax proteins. Blood 87: 3410-7.

37. Nakayama Y, Ishikawa C, Tamaki K, Senba M, Fujita J, Mori N (2011) Interleukin-1 alpha produced by human T-cell leukaemia virus type I-infected T cells induces intercellular adhesion molecule-1 expression on lung epithelial cells. J Med Microbiol 60: 1750-61.

38. Sagawa K, Mochizuki M, Katagirl K, Tsuboi I, Sugita S, et al. (1996) In vitro effects of immunosuppressive agents on cytokine production by HTLV-Iinfected T cell clones derived from the ocular fluid of patients with HTLV-I uveitis. Microbiol Immunol 40: 373-9.

39. Kurihara K, Harashima N, Hanabuchi S, Masuda M, Utsunomiya A, et al. (2005) Potential immunogenicity of adult $\mathrm{T}$ cell leukemia cells in vivo. Int J Cancer 114: 257-67.

40. Takeda S, Maeda M, Morikawa S, Taniguchi Y, Yasunaga J, et al. (2004) Genetic and epigenetic inactivation of tax gene in adult T-cell leukemia cells. Int J Cancer 109: 559-67.

41. Wano Y, Hattori T, Matsuoka M, Takatsuki K, Chua AO, et al. (1987) Interleukin 1 gene expression in adult T cell leukemia. J Clin Invest 80: 911-6.

42. Elaraj DM, Weinreich DM, Varghese S, Puhlmann M, Hewitt SM, et al. (2006) The role of interleukin 1 in growth and metastasis of human cancer xenografts. Clin Cancer Res 12: 1088-96.

43. Saijo S, Asano M, Horai R, Yamamoto H, Iwakura Y (2002) Suppression of autoimmune arthritis in interleukin-1-deficient mice in which $\mathrm{T}$ cell activation is impaired due to low levels of CD40 ligand and OX40 expression on T cells. Arthritis Rheum 46: 533-44.

44. Roberti MP, Rocca YS, Amat M, Pampena MB, Loza J, et al. (2012) IL-2- or IL-15-activated NK cells enhance Cetuximab-mediated activity against triplenegative breast cancer in xenografts and in breast cancer patients. Breast Cancer Res Treat 136: 659-71.

45. Luedke E, Jaime-Ramirez AC, Bhave N, Rhoda J, Choudhary MM, et al. (2012) Cetuximab therapy in head and neck cancer: immune modulation with interleukin-12 and other natural killer cell-activating cytokines. Surgery 152: 431-40.

46. Moga E, Cantó E, Vidal S, Juarez C, Sierra J, Briones J (2011) Interleukin-15 enhances rituximab-dependent cytotoxicity against chronic lymphocytic leukemia cells and overcomes transforming growth factor beta-mediated immunosuppression. Exp Hematol 39: 1064-71.

47. Zhang M, Yao Z, Dubois S, Ju W, Müller JR, Waldmann TA (2009) Interleukin-15 combined with an anti-CD40 antibody provides enhanced therapeutic efficacy for murine models of colon cancer. Proc Natl Acad Sci U S A 106: 7513-8.

48. Zhang M, Ju W, Yao Z, Yu P, Wei BR, et al. (2012) Augmented IL-15R $\alpha$ expression by CD40 activation is critical in synergistic CD8 T cell-mediated antitumor activity of anti-CD40 antibody with IL-15 in TRAMP-C2 tumors in mice. J Immunol 188: 6156-64.

49. Weiss JM, Back TC, Scarzello AJ, Subleski JJ, Hall VL, et al. (2009) Successful immunotherapy with IL-2/anti-CD40 induces the chemokine-mediated mitigation of an immunosuppressive tumor microenvironment. Proc Natl Acad Sci U S A 106: 19455-60. 
50. Hong DS, Naing A, Falchook GS, Piha-Paul S, Wheler JJ, et al. (2011) Abstract A211: A phase I study of MABp1, a first-in-human, first-true human monoclonal antibody against the IL-1 in patients with advanced cancers. Molecular Cancer Therapeutics 10: Supplement 1. doi: 10.1158/1535-7163. TARG-11-A211. 